\title{
$\mathfrak{I} \mathfrak{H} \mathfrak{g} \mathfrak{E} \mathfrak{n} \mathfrak{d} \mathfrak{l} \mathfrak{x} \mathfrak{h} \mathfrak{x} \mathfrak{E}$
}

\author{
(fin Burt)
}

fuiir

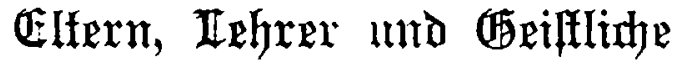

noit

Ir. W. Inerftex

106. bia 110. TauTend

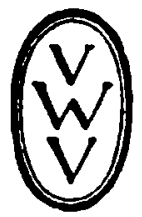

Bexlin und Triptig 1922

Dexeintgung wi

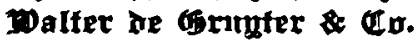

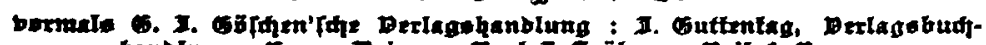

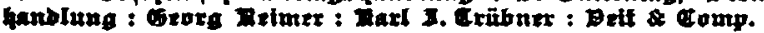


Hule Pichte, inçbeiondere bas ber über: jegung in fremne Enrahen, norbehalten.

Copyright Iq12 by Georg Reimer 
Dem ग्र̆แDenken

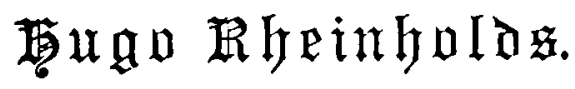





\section{Orftes Bormort.}

Die borliegenbe $\mathscr{A r b e i t}$ ift unmittelbar aus ben Inregungen ber

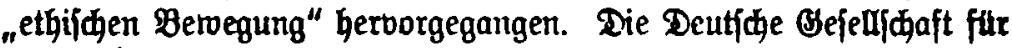

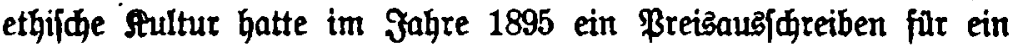

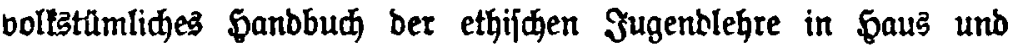
Shule erlaffen. (5s follte mit einem foldhen Bude inmitten bes Stampfes ber Beltanjajaungen eine Bufammenfaffung und ETlăuterung berjenigen Brtinde bes Sittlidjen gegeben werben, bie unabhängig bon ben trennenben $\mathfrak{A} n \mathfrak{i}$ 西ten aber bie legten Dinge unb banm für bie gemeinfame ethif Zugendlefre in ber bffentliden Squle bon ganz befonberer Bebeutung finb. Uud rutbe barauf hingewiejen, baß es angefichts ber Entfrembung breiter Boltasidichten von Religion unb Airche, forvie angejidts ber tritifhen Stimmung bes Beitalters gegenuber aller 2 rabition, bringend notwenbig fei, ber Jugenb neben

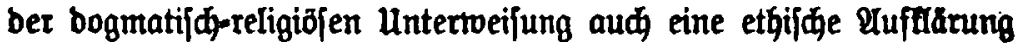
unb Anregung zuteil werben zu laffer, bie lebiglid an bie unmittelbar einleudtende Lebenabeobactung und Selbfterfahrung appelliert.

Der Berfaffer hat fid bamals an ber geftellten Aufgabe nidjt beteiligt, wohl aber feit bem Jahre 1897 in 8 thrid regelmäfig ethifde Sturfe fur Anaben und Mäbden beridiebener 2atersfufen beranftaltet. Aus ben Erfahrungen biefes Unterridtes ift allmăblid bas vorliegenbe Buథ mit feinen Beifpielen unb Folgerungen entfanden. Es möhte bagu anregen, auf allen Sebieten ber Sugenbfeelforge - alfo nidt nur in Sujule, इaus unb Sirdhe, fonbern aud in Storrettionsanftalten, Befängniffen, Sntematen, Stinberhorten $x$. - bie Brunblage ber etgifden Eintirtung breiter unb tiefer zu legen.

Ic megr bie Bewegung gegen bie torperlidge 8urhtigung ber Stinber zunimmt unb bamit ben Ergieger von ăußerliळen Dişziplinarmitteln auf eine mebr innerlide Beeinfluffung bertweift, um fo brin- 
genber wirb ez and, bieje innerlide Beeinfluffung zu einem $b e$. fonberen segenitand ber pabagogilden Bor. bereitung unb 5 dulung zu ergeben.

Benn ber Berfaffer in ben von ihm gebradten Beifpielen auz.

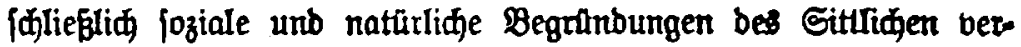
trettet unb ben שppell an religiōje Borfellungen und Befüble vermeibet, fo entfpridt bas ber bejonbeten Aufigabe feines Butjes. - (ar

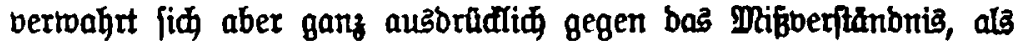
ftehe er bamit auf bem Boben jener Mabitalen, welde in ETziehung und Leben bie Meligion burd bie blope Moral exfezen toollen.

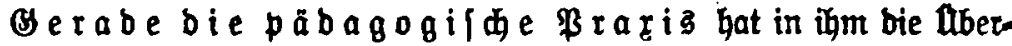

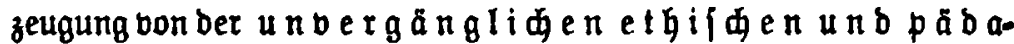
bogifden Bebeutung ber Religion a f bo 由fte berfärt. Jn ber offentliden Sdule ift ja allerbings ein obligatorifder Meligionsunterridt auf bie Dauer nidt haltbar, rwegen ber babei unbermeibliden Bergewaltigung anberg benlenber Gitern - eine tonfeffionell neutrale Sittenlebre ift hier wohl

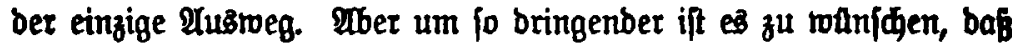
bann aukerhalb ber Sxule fur Raum und Bielegenheit zu religibjer Ergånzung unb Bettiefung ber Sittenlehre gejorgt werbe. Der Ber. faffer hofft jogar, baß gerabe bas borliegente Bud religiōs gleidgultige Gitem in bezug auf bie frembaltung ihrer Sinber bon religioben Ginfluffen etroas nabbentlidier maßjen twerbe.

Finem Ginwambe foll gleid an biejer Stelle begegnet werben:

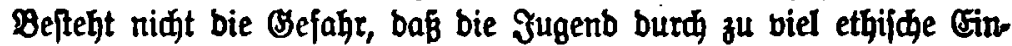
wirtung ihrer Frijde unb unrpringlidfeit beraubt recte? Stun ber Lejer wirb jehen, baß̉ bas borliegenbe Buch gerabe biejer Befajs begegnen will, inbem Borjhläge gibt, wie man an Stelle zu vielen

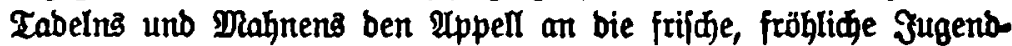
traft in ben Borbergrunb ber ethildhen Unregung ftellen tonne. $\mathbf{3}$ ubrigen ift es Tatfache, bak stinber, welde ohne tiefere Bildoung bes

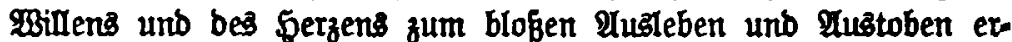

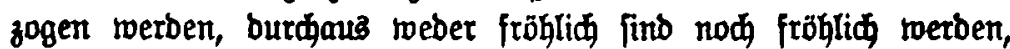
jonbern mürrifめ unb reizbar - eben reil fie auf Satitt unb Tritt in Streit unb Banl geraten unb roeber mit ben fleinen now mit ben

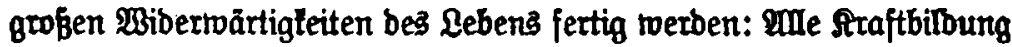
madt froh und alles Sidgebenlajfen madt elenb unb unzufrieber. 


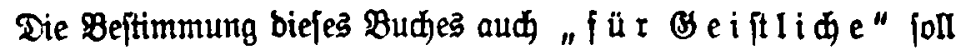
teine anmaßenbe Ginmijđung in bie religiője Seeljorge bebeuten. Der Berfaffer glaubt einem ihm mehrjach bon geiflidaer Seite geäußerten Bebürinis zu entfprehen, wenn er fpeziell bem Scelforger in (Brofifäbten einiges Material für bie angewandte Sittenlehre zur Berfügung ftellt.

Es fei nod, bemertt, bak in ben Beifpielen abfidtlid ber ztwangloje Ton ber Bciprechung feitgehalten worben ift; Wieberholungen bes Shemas ließen fid nidt immer vermeiben, oa ber Berfalfer bem LeGrer für beftimmte Cinmirłungen mo̊glidfit mannigfaltige Bartationen zur Berfilgung fillen rollte. Die Beifpiele find für Inaben unb Mäbden von 11-15 ̧ahren gebadit und erfdeinen mit wenigen Qluąnahmen unter bem Titel "Lebenth bireft far ben Bebraud ber Jugend.

Butid, ben 1. Mai 1904.

\section{Der Berfaffer.}

\section{3'weite Borwort.}

Seit bem Erffjeinen bes borfiegenben Buches finb nun fapt brei Jahre verfloffen. Es liegt nahe, baß ber Berfaffer bie Belegenheit eines Reubrud benukst, um gegenüber ben Beurteilungen, bie fein Bud in ben verfdiebenen Ragern gefunden hat, nod einmal hurz bie Grumbanfdaunng zu betonen und zu verteibigen, von ber feine päbagogijめe $\mathfrak{A}$ rbeit getragen roirb.

Die faft einmultige Buftimmung ganz entgegengejefter Midytungen hat mohl beutlid berwiejen, wie widtig es ift, getabe in bem Beitalter ber leiben[daftlidfiten Blaubenstrennungen an einen nod borhanbenen gemeinjamen Befib bon etbifden Intereffen anzufnitpfen unb bie Menifhen bon bort aus zu jener Selbiterfenntnis unb lebenz. fenntniz zurüđzuleiten, bie allein vieber ein tieferes Berftänonis ber Meligion möglid maden farn. Solder Dethobil finb heute alle ftreitenben ßartei» gleid bedürftig: bie Blaubenalojen, weil fie aus Mangel an $\mathfrak{B}$ ertief u $\mathrm{g}$ in bie Brunbfragen ber ethifden $\mathrm{Er}_{\text {. }}$ 
ziehumg unb Selb/tergiegung bie Meligion gar nidgt megr berftegen:

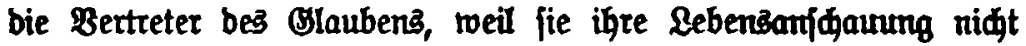
mehr lebenbig barzuftellen bermögen, zu wenig von ber einfadfiten etbifden Exfahrung oulgeben, zu jebr bebultio unb zu wenig inbultio lebren und interpretieren.

Wäre auf religiőjer Seite biefer leitenbe (s) $\mathrm{ef}$ i Buđ̆es mehr beađtet unb herausgefühilt worben, fo hätten mandie Sritifer nibt bie allzu geringe Betonung unb Bebanblumg bes Meli-

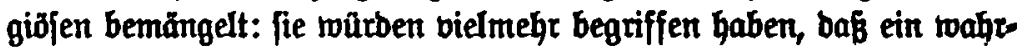

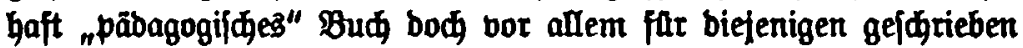
werben mus, bie aberzeugt unb weitergefuhnt werben follen - nidjt blok far biejenigen, weldge bereits thergeugt finb unb anbertoärţ

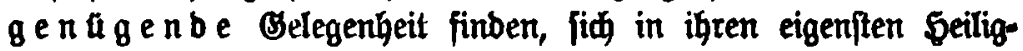
tilmern zu befeftiger.

Bon freibenterif wer Seite ift lebhaft. bellagt worber, baß bas Puळ von einem fo entidiebenen Betenntrib zu ber unerreid. baren päbagogifłen Straft ber driftliden Neligion getragen ift. Der

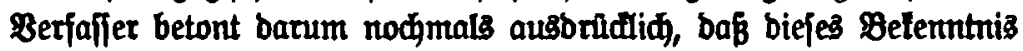
nidt aus einer beliebigen metaphbfifden saune, fondern gerabe aus bem Sern feiner moralpäbagogifden Stubien

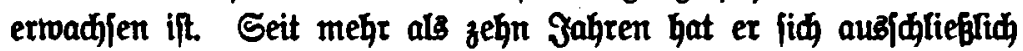

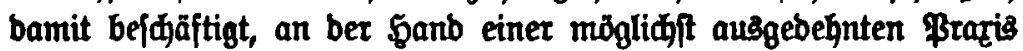
ber ethifhen Jugenbunterweifung bos Problem ber Oharalterbiloung pfydologifक zu fubieten unb burdyzubcnten - bas Ergebnis biefer

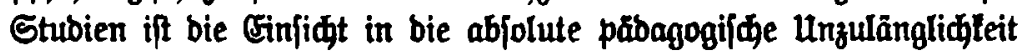
aller religionsilofen Sugenberziegung *). unb ber Berfaffer ift aber-

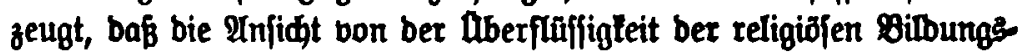
mittel nur beşalb jo weite streije mobemer \$äbagogen zu bebertiden bermag, weil biejelben auf bem (s) ebiete ber çaralterpãbogogil feine auzgeberjnte ßraris, tein augjhließlides umo Ionzentrierte Stubium hinter fid haben. Ouf biefen $\mathfrak{u m p t a n b}$ muß um ber Sadje trillen ausbrattid hingetwiejen werben - weil hier bon philofophifden Sdifrifttellem und bon blo

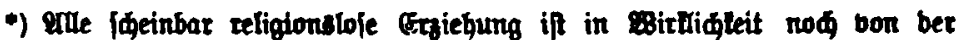
Ma Exf in ber tommenben cenemtion witb man beutliger effahter, was eigentfid refigionstoje Griebung bebeutet. 


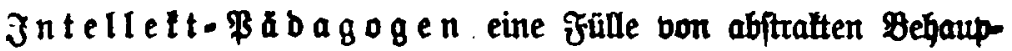
tungen aufgeftellt worben finb, bie mit ben trirtliden Iatfađjen unb Beburfniffen bet menjditiden Natur in grellem Miberfprud fteben. fein Proralgebot bermag bie Seele bes Menjळen fo in igrer eigeniten

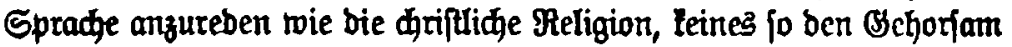
mit ber Befreiung bas "Stirb" mit bem "MBerbe", bas Dpfer mit ber Âuferftebung zu bermählen. 23irb bies enf mieber erfannt werben, jo wirb man einfehen, baß́ bie religiőle Begrunoung ber ctthil nidgt

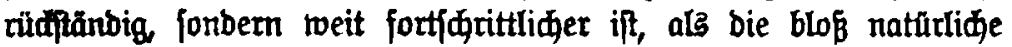
Sittenlegre - eben weil jene bas äußere Bebot in bas perionlidifite \&eben zu über $[$ eken weiß uno barum bas überwinbet, was ßaulus bie "Inedtjdaft bes Befezes" nennt und was ber Moral gerabe bei

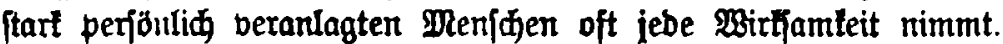

Iuf ber anberen Seite muß aud mandien einjeitigen Bertretern ber firdlidgen Bäbagogif gejagt roerben, baß̧ bie majeftätifdbe Be= bärbe, mit ber fie bie ethijden Beftrebungen freigefinnter Atreife ablegner, nidt ber unbeftreitbaren Bebeutung gerecht wirb, welde biefe Beftrebungen in ber getwaltigen stulturftife ber Begentwatt Gaber. Infang einer Studfehr zur Innentultur. Dies jolte nidt therjełen unb niđt geringldäz̧ig behanbelt werben. In bielen mobernen Sulturzentren roadfen boch bie bon ber stirdie abgefallenen Boltstreife allmăhlid zu Dlajorităten an. Der geiftigen sage biefer 2tbgefallenen with aber weber burd einen erzroungenen Feligionsuntertidht, nod burd ben bloken abftralten Sinneis auf bie Armut ber religions.

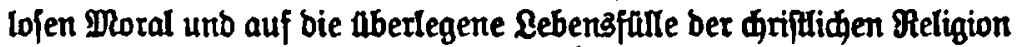
ridtig geholfen. Dent wenn nidt ftatt biejer \&ebensfüle po erfidredent viel $\mathfrak{A}$ rmut an wirllibem Leben gerabe aus in ber religiofen unterweifung toäre, fo hätte man wohl niđht eine fo groß̉e Bahl bon $2 \mathfrak{b}$. gefallenen zu betlagen. DRan rebibiere baber auf religiöjer Seite bor allem bie eigene Methobe, bamit fie wahthaft Beugnis ablege von ber feelenbilbenben und feelengetwinnenten ftraft ber Feligion, unb man ante im abrigen alle jene ethifhen Beftrebungen zunäbft als erfte Sdititte ber $\mathfrak{u m}$ lebr von aupen nad innen; man betradte fie ferner aud als unentbehrlide neutralc Methoben inmitten

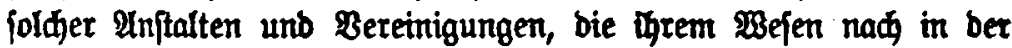
(Slaubensfrage niøt Partei nehmen burfen. gu betämpfen braudat 
man fie nur bort, wo fie aus ibrer prattifकen Befdränlung auf rein ethif

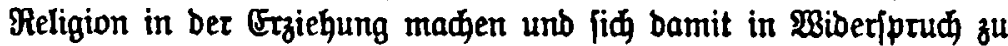
ber Erfahrung aller Gahrhunderte fezen.

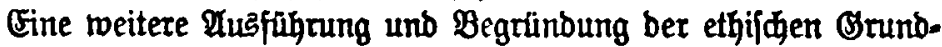
gebanlen bes borliegenden Budes foll in einem weiteren Buble gegeben werben, bas unter bem Titel "Rebensführung" im Raufe biejes Sahres erjळeinen wirb. Bur Beantwortung vielfadjer Einwänbe gegen ben religiōjen Stanbpunlt bes Berfaffers wirb - ebenfalls im Saufe bes gahres - eine Stubie über " Religion und đharalterbilbung" erfdeinen').

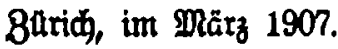

ชr. 23. Foerfiter.

\section{Dritte Bormort.}

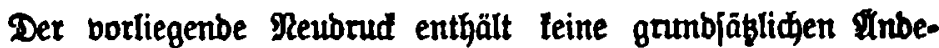
numger. Start umgearbeitet umb erweitert murben mur bie Quto fuhrungen aber bie päbagogilðe Behanblung ber \&uge (S $316 f f$.). Der Beridt thber bie Lage bea ethijhen unterridts in Englanb wurbe Durd Datteilungen über widtige neuere Entwiftungen ergānżt (5. 190). Cnolid wurben in bas Sapitel über bie Befrafung ber Pinber einige 2ntwenbungen auf bie Praris ber 3ugenbgeridte eingefungt (S. $700 \mathrm{ff}$.).

8üriđ, im Mai 1909.

g̊. Foerfter.

1) Die Fublifation biefer Stubie nod ver/doben worben, ba ber Bexfaffer biefelbe ju einer pringipiellen auterjudung erwettern will. 


\section{Bierte $\mathfrak{B a r w o r t .}$}

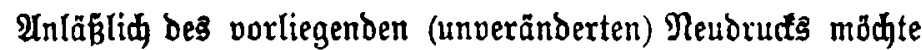
ber Berfaffer ganz furz bie IIngriffe berühren, bie neuerbiugs von ganz beftimmter, firdliđjer Seite gegen feine Jugendlegre geridtet worben fint ${ }^{1}$ ): $\mathfrak{M a n}$ ftellt mit vollem Recte feft, bas jents Buth, wo es religiöje ßrobleme berührt, vielfach nod Spuren einer Denlfweife zeigt, bie der $\mathfrak{B e r f a j f e r ~ f p a ̈ t e r ~ u ̈ b e r i v u n d e n ~ h a t ; ~ f o ~ f i n d ~}$ bie driftliden Dogmen mehrfac rein inmbolij内 gebeutet, was meiner jegizen 2luffaffung nidt mehr entipridt uno wons aud $b a=$ ntals feinesiwega als eridöpfende Deutung, jondern nur als ein 3ugang für bie ber Meligion entjrembeten Sreife gedadjt war. Diejes ftelle id biermit ausbrüfflid nodmals feft, ba imnter wieder

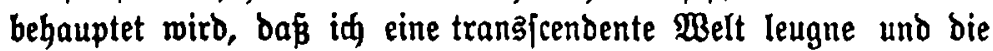

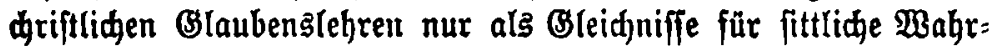
beiten annebme. Iめ babe mid in meinen ppäteren Sdjriften un= zreibeutig zur Tranşcendenz betannt; wenn id bie Jugendlehre noch nidit entiprechend umgearbeitet habe, fo gefdah bas nidft nur, weil mir bişher bie Zeit zu einer vollftänbigen Umarbeitung gefehlt bat, fondern aud, weil id jeneş Bud, als ein labergangsbud, nit allen darafteriftifden Beiden einer 3 wijhenftufe befteben laffen wollte und es vorzog bas Crgänzende und Storrigierende in neuen Büdern Dargulegen. Biele Rritiler haben, wie es \{deint, bie Borworte ber Jugendlegre nidt gelefen und baher etwas gefuct,

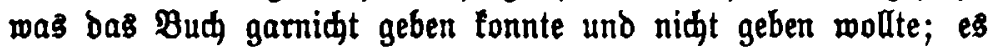
hat fich aubbrüctid auf ben Ippell an bie natürlicen Motive bef̧ränft - bieje Bejđeibung enthưlt aber feineşwegs eine Gering[đäzung ber überragenden Bebeutung bes religiöjen Elements,

1) Näberes finbet fth in folgenben @đriften des Berfafiers: 1. Chriftentum unb Bäbagogit, eine Iuseinanberję̧ung mit Đerm Dom: betan Dr. Rief. 2. Mein Sdlufwort an \$errn Dombelan Dr. Rief, Sonberaborud ber Ratedetijめen Blătter \$eft 6-8, 1920, Roefels Berlag Seinpten. 
fonbern bebeutete nur eine Bejărănhung auf biejenigen praftijळen Crfabnungen, bie ber Berfaffer auf einen ganz beftimmten Bebiete gefammelt hat und bie mit einem Teilelement ber Crziebung zu tun haben, bas für bie moberne Ĵugenbjeeljorge zweifellos von fleigender propäbeutifalder Bidtigleit ift.

Büriđ, Djtern 1921.

8ร. 2 B. Foerpter. 